\title{
Clinical chemistry score versus high-sensitivity cardiac troponin I and T tests alone to identify patients at low or high risk for myocardial infarction or death at presentation to the emergency department
}

\author{
Peter A. Kavsak PhD, Johannes T. Neumann MD, Louise Cullen MBBS PhD, Martin Than MBBS, \\ Colleen Shortt PhD, Jaimi H. Greenslade PhD, John W. Pickering PhD, Francisco Ojeda PhD, Jinhui Ma PhD, \\ Natasha Clayton CRA RA, Jonathan Sherbino MD, Stephen A. Hill PhD, Matthew McQueen MBChB PhD, \\ Dirk Westermann MD, Nils A. Sörensen MD, William A. Parsonage MBBS DM, Lauren Griffith PhD, \\ Shamir R. Mehta MD MSc, P.J. Devereaux MD PhD, Mark Richards MBChB PhD, Richard Troughton MBChB PhD, \\ Chris Pemberton PhD, Sally Aldous MBChB MD, Stefan Blankenberg MD, Andrew Worster MD MSc
}

Cite as: CMAJ 2018 August 20;190:E974-84. doi: 10.1503/cmaj.180144

\begin{abstract}
BACKGROUND: Testing for high-sensitivity cardiac troponin (hs-cTn) may assist triage and clinical decision-making in patients presenting to the emergency department with symptoms of acute coronary syndrome; however, this could result in the misclassification of risk because of analytical variation or laboratory error. We sought to evaluate a new laboratorybased risk-stratification tool that incorporates tests for hs-cTn, glucose level and estimated glomerular filtration rate to identify patients at risk of myocardial infarction or death when presenting to the emergency department.
\end{abstract}

METHODS: We constructed the clinical chemistry score (CCS) (range 0-5 points) and validated it as a predictor of 30-day myocardial infarction (MI) or death using data from 4 cohort studies involving patients who presented to the emergency department with symptoms suggestive of acute coronary syndrome. We calculated diagnostic parameters for the CCS score separately using high-sensitivity cardiac troponin I (hs-cTnl) and high-sensitivity cardiac troponin $\mathrm{T}$ (hs-cTnT).

RESULTS: For the combined cohorts ( $n=$ 4245), $17.1 \%$ of participants had an MI or died within 30 days. A CCS score of 0 points best identified low-risk participants: the hs-cTnl CCS had a sensitivity of 100\% (95\% confidence interval [Cl] 99.5\%-100\%), with $8.9 \%$ (95\% Cl 8.1\%-9.8\%) of the population classified as being at low risk of $\mathrm{MI}$ or death within 30 days; the hs-cTnT CCS had a sensitivity of $99.9 \%(95 \% \mathrm{Cl} 99.2 \%-$ $100 \%$ ), with $10.5 \%$ (95\% Cl 9.6\%-11.4\%) of the population classified as being at low risk. The CCS had better sensitivity than hscTn alone (hs-cTnl < $5 \mathrm{ng} / \mathrm{L}: 96.6 \%, 95 \% \mathrm{Cl}$ 95.0\%-97.8\%; hs-cTnT < 6 ng/L: 98.2\%,
95\% Cl 97.0\%-99.0\%). A CCS score of 5 points best identified patients at high risk (hs-cTnl CCS: specificity $96.6 \%, 95 \% \mathrm{Cl}$ 96.0\%-97.2\%; $11.2 \%$ [95\% Cl 10.3\%$12.2 \%$ ] of the population classified as being at high risk; hs-cTnT CCS: specificity 94.0\%, 95\% Cl 93.1\%-94.7\%; 13.1\% [95\% $\mathrm{Cl} 12.1 \%-14.1 \%]$ of the population classified as being at high risk) compared with using the overall 99th percentiles for the hs-cTn assays (specificity of hs-cTnl 93.2\%, 95\% Cl 92.3-94.0; specificity of hs-cTnT $73.8 \%, 95 \% \mathrm{Cl} 72.3-75.2)$.

INTERPRETATION: The CCS score at the chosen cut-offs was more sensitive and specific than hs-cTn alone for risk stratification of patients presenting to the emergency department with suspected acute coronary syndrome. Study registration: ClinicalTrials.gov, nos. NCT01994577; NCT02355457

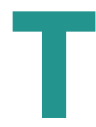

he use of a single high-sensitivity cardiac troponin (hscTn) test concentration on presentation to the emergency department has been proposed for triage and early decision-making in patients with suspected acute coronary syndrome. ${ }^{1-3}$ However, a single low concentration result for hscTn for identifying patients at low risk in the emergency department may misclassify patients, because an analytical variation of $\pm 3 \mathrm{ng} / \mathrm{L}$ for the hs-cTn assays at the low analytical range is 
acceptable; yet, this variation will reclassify patients using the published algorithms. ${ }^{4-6}$ In practice, different lots of reagents for both hs-cTnl and hs-cTnT have already been observed to yield variation that exceeds $3 \mathrm{ng} / \mathrm{L} .{ }^{7,8}$ In addition, there are several interferences that affect hs-cTn measurements. ${ }^{9,10}$ Together, this necessitates improved laboratory health services to aid physicians in evaluating patients with possible acute coronary syndrome.

We have previously assessed the utility and cost-effectiveness of combining assessment of the glucose level with hs-cTn concentrations for diagnosing myocardial infarction (MI) in the emergency department. ${ }^{11,12}$ While this dual-marker approach was cost-effective compared with hs-cTn alone, it was found to be suboptimal for short-term risk stratification. For risk stratification based on laboratory services to be accepted into practice, it must be easy to implement and involve only validated laboratory tests that are widely and routinely measured in patients presenting with symptoms of acute coronary syndrome and also safe.

In an effort to have a robust laboratory service to aid physicians and reassure patients in the emergency department setting, we have developed a laboratory medicine test score using common clinical chemistry tests (i.e., a clinical chemistry score [CCS]) for MI or death at $30 \mathrm{~d}$ ). This score encompasses the measurement of hs-cTn concentration, glucose level and estimated glomerular filtration rate (eGFR), with eGFR also providing important prognostic information in this setting. ${ }^{13}$ Specifically, for ST-segment elevation MI (STEMI), high glucose concentrations at admission to the emergency department identify patients who are more hemodynamically unstable, have a larger infarct size and have a high 30-day mortality. ${ }^{14}$ Moreover, eGFR calculated by the CKD-EPI creatinine equation has been shown to be an independent predictor of major adverse cardiac outcomes in patients with acute coronary syndrome. ${ }^{15}$ We previously assessed the utility of hematologic variables in addition to the clinical chemistry tests. ${ }^{16}$ However, as our goal was to develop a simple algorithm that could be validated externally across different continents, we decided to limit the algorithm to these 3 clinical chemistry tests that are available in most populations of patients with chest pain in the emergency department. A recent publication using machine learning for predicting 30-day mortality after STEMI ranked creatinine and glucose among the top 3 variables (and top 2 laboratory tests) using classifier-based machine-learning algorithms, ${ }^{17}$ which further supports the inclusion of eGFR, glucose and hs-cTn in assessing patients with possible non-ST-elevation acute coronary syndrome.

In the present study, we tested and assessed the CCS in patients without STEMI who presented to the emergency department, across different populations from North America, Europe and Australasia, to determine if score thresholds resulted in sufficient sensitivity and negative predictive value (NPV) to safely identify patients at low risk. We assessed whether the CCS could achieve a sensitivity greater than $99.0 \%$ for MI or death at 30 days as this is an estimate that most physicians in the emergency department consider as safe for discharge. ${ }^{18}$ We also evaluated whether the score had sufficient positive predictive value (PPV) and specificity to identify patients at high risk who may benefit from early management.

\section{Methods}

\section{Study design and participants}

Validation of the performance of the CCS test was a post hoc analysis performed in 4 emergency department populations in Hamilton, Ontario, Canada (Hamilton General Hospital and Juravinski Hospital, Hamilton Health Sciences, and St. Joseph's Healthcare Hamilton); Brisbane, Australia (Royal Brisbane and Women's Hospital); Christchurch, New Zealand (Christchurch Hospital); and Hamburg, Germany (University Hospital HamburgEppendorf, University Heart Center Hamburg). Briefly, patients in the emergency department who were enrolled in all 4 studies had symptoms suggestive of acute coronary syndrome (patients with STEMI were excluded), with measurement of both glucose and creatinine levels at presentation as part of routine care (Appendix 1, available at www.cmaj.ca/lookup/suppl/ doi:10.1503/cmaj.180144/-/DC1; additional details of the study methods, Appendix 2, available at www.cmaj.ca/lookup/suppl/ doi:10.1503/cmaj.180144/-/DC1). ${ }^{19-23}$

\section{Health outcomes}

The primary outcome was 30-day $\mathrm{MI}^{24}$ or all-cause death. Secondary outcomes included index MI, 30-day revascularization rate (percutaneous coronary intervention or coronary artery bypass graft) and 30-day composite of unstable angina, MI or allcause death (values for unstable angina were not available for the Hamburg cohort) (Appendix 2).

\section{Assay methods and clinical chemistry score}

Standard acceptable laboratory methods were used to measure glucose and creatinine levels (used to calculate eGFR). Both the Roche Elecsys Troponin T hs and Abbott ARCHITECT High Sensitive Troponin-I assays used in these studies met the most recent laboratory recommendations for total analytic error for hs-cTn assays $^{6}$ (Appendix 2).

The approach to developing the CCS was pragmatic, using equal weighting for eGFR and glucose, and using established and recognized cut-offs for normality for these tests. For hs-cTn, the weighting was based on the graded risk and on analytical performance characteristics of the assays. Briefly, the CCS included glucose, eGFR and hs-cTnT or hs-cTnl (i.e., CCS with hs-cTnT or CCS with hs-cTnl, respectively, as clinical laboratories will typically report only one hs-cTn test) (Box 1). The cut-offs used for each test were based on accepted normal levels and low-risk clinical cut points, with values of eGFR of $90 \mathrm{~mL} / \mathrm{min} / 1.73 \mathrm{~m}^{2}$ or more, glucose of less than $5.6 \mathrm{mmol} / \mathrm{L}$, hs-cTnl of less than $4 \mathrm{ng} / \mathrm{L}$ and of hs-cTnT less than $8 \mathrm{ng} / \mathrm{L}$ used to identify patients at low risk (i.e., abnormal value 1 point and normal 0 points). ${ }^{13,25-27}$ For hs-cTn, 2 additional cut points were selected based on analytical variation, population and health outcome studies, ${ }^{26-32}$ to have a final CCS range of $0-5$ points (i.e., sum of the points for the results for glucose, eGFR and hs-cTn obtained from the patient's 
Box 1: Clinical chemistry score

\section{CCS with hs-cTnT}

Glucose

- $<5.6 \mathrm{mmol} / \mathrm{L}=0$ points

- $\geq 5.6 \mathrm{mmol} / \mathrm{L}=1$ point

eGFR

- $<90 \mathrm{~mL} / \mathrm{min} / 1.73 \mathrm{~m}^{2}=1$ point

- $\geq 90 \mathrm{~mL} / \mathrm{min} / 1.73 \mathrm{~m}^{2}=0$ points

hs-cTnT

- hs-cTnT $<8 \mathrm{ng} / \mathrm{L}=0$ points

- hs-cTnl 8-18 ng/L = 1 point

- hs-cTnl 19-30 ng/L = 2 points

- hs-cTnl $>30 \mathrm{ng} / \mathrm{L}=3$ points

Sum of glucose, eGFR and hs-cTnT

CCS range: $0-5$ points

\section{CCS with hs-cTnl}

Glucose

- $<5.6 \mathrm{mmol} / \mathrm{L}=0$ points

- $\geq 5.6 \mathrm{mmol} / \mathrm{L}=1$ point

eGFR

- $<90 \mathrm{~mL} / \mathrm{min} / 1.73 \mathrm{~m}^{2}=1$ point

- $\geq 90 \mathrm{~mL} / \mathrm{min} / 1.73 \mathrm{~m}^{2}=0$ points

hs-cTnT

- hs-cTnl $<4 \mathrm{ng} / \mathrm{L}=0$ points

- hs-cTnl 4-14 ng/L = 1 point

- hs-cTnl 15-30 ng/L = 2 points

- hs-cTnl $>30 \mathrm{ng} / \mathrm{L}=3$ points

Sum of glucose, eGFR and hs-cTnl

CCS range: $0-5$ points

Note: $\mathrm{CCS}=$ clinical chemistry score, eGFR = estimated glomerular filtration rate, hs-cTn = high-sensitivity cardiac troponin

blood work at presentation to the emergency department; Supplemental Table 1, Appendix 3, available at www.cmaj.ca/ lookup/suppl/doi:10.1503/cmaj.180144/-/DC1).

\section{Statistical analyses}

We first tested the CCS algorithm in the Hamilton cohort for risk stratification, with the expectation that a CCS closer to 0 points (all laboratory results were normal) would be suggestive of low risk (with scores that yielded about a $1 \%$ outcome rate evaluated further) and a CCS closer to 5 points would be suggestive of high risk (all laboratory results were abnormal with scores that yielded about a 50\% outcome rate evaluated further). First, we used test parameters (sensitivity and specificity) to identify possible CCS cut-offs for 30-day MI and death via Kaplan-Meier survival analysis, and then the Cox proportional hazards model to provide hazard ratios (HRs; adjusted for age, sex, family history, hypertension, hypercholesterolemia, diabetes mellitus and smoking status $)^{19}$ to support the selected cut-offs. The selected CCS cutoffs were then applied to the other 3 population cohorts to assess sensitivity, specificity, PPV, NPV and likelihood ratios. In this regard, we provided the CCS algorithm to each site to determine the test performance and descriptive statistics for their emergency department cohort.

We calculated the sensitivity, specificity, PPV, NPV and likelihood ratios with 95\% confidence intervals (Cls) for each cohort as well as for the combined population (by adding the number of participants in each cohort) for the low- and high-risk CCS cutoffs. We performed receiver operating characteristic (ROC) curve analysis for the CCS algorithms for each emergency department population, including sex-specific analysis that assessed the CCS algorithms for the primary outcome, with comparison to hs-cTn concentration alone (low risk: hs-cTnl $<5 \mathrm{ng} / \mathrm{L}$ or hs-cTnT $<6 \mathrm{ng} / \mathrm{L}$; high risk [overall 99th percentiles]: hs-cTnl $>26 \mathrm{ng} / \mathrm{L}$ or hs-cTnT > $14 \mathrm{ng} / \mathrm{L})^{3,30,33}$ in the overall emergency department population. We generated the ROC curves based on data without smoothing, and we calculated the area under the ROC curves (AUC) correspondingly. We performed the statistical analysis using SAS 9.4, MedCalc statistical software, Graphpad prism, R3.2.2 (R Project for Statistical Computing) and StatsDirect statistical software.

\section{Ethics approval}

This study was approved by the Hamilton Integrated Research Ethics Board, the Royal Brisbane and Women's Hospital Human Research Ethics Committee, the New Zealand Health and Disability Ethics Committee, and the local ethics board of Hamburg (Ethics committee of the Ärztekammer Hamburg, Germany). We followed the Standards for the Reporting of Diagnostic Accuracy Studies guideline.

\section{Results}

We included 4245 participants in this study, with 727 (17.1\%) having the primary outcome (30-d MI or all-cause death). The prevalence of the primary outcome was different between the cohorts $(p<0.001)$, with the Brisbane cohort $(n=795)$ having the lowest prevalence at $6 \%$, followed by the Hamilton cohort $(n=1092)$ at $16 \%$, the Hamburg cohort $(n=1412)$ at $20 \%$, and the Christchurch cohort at 24\% (Table 1). A higher percentage of males were enrolled at the Brisbane, Christchurch and Hamburg sites $(\geq 60 \%$ of the 3 cohorts) compared with the Hamilton site ( $<50 \%$ males). Nearly $30 \%$ of participants in the Hamilton cohort had diabetes compared with less than $20 \%$ in the 3 other cohorts.

In the Canadian test population (Hamilton), the lower the CCS, the lower the rates of MI or death over 30 days (i.e., the primary outcome, Supplemental Figure 2, Appendix 1). In the Hamilton cohort, a CCS value less than 1 yielded $100 \%$ sensitivity, less than 2 yielded a sensitivity of $99 \%$ and less than 3 a sensitivity of 93\% for the primary outcome using either hs-cTnl or hs-cTnT (Supplemental Figure 3, Appendix 1). A CCS of 4 points or more yielded a specificity of $85 \%$ with hs-cTnl and $76 \%$ with hs-cTnT, whereas a CCS of 5 points yielded a specificity of $96 \%$ with hscTnl and $90 \%$ with hs-cTnT. Participants with a CCS of 5 points, reflecting abnormal results for all laboratory tests, represented a high-risk group, with an adjusted HR for patients with a CCS of 5 points versus those with a CCS of less than 5 points of 4.63 
Table 1: Participant characteristics

\begin{tabular}{|c|c|c|c|c|}
\hline Characteristic & $\begin{array}{l}\text { No. }(\%) \text { of } \\
\text { participants* in the } \\
\text { Hamilton cohort } \\
n=1092\end{array}$ & $\begin{array}{c}\text { No. }(\%) \text { of } \\
\text { participants }{ }^{\star} \text { in the } \\
\text { Brisbane cohort } \\
n=795\end{array}$ & $\begin{array}{l}\text { No. }(\%) \text { of } \\
\text { participants }{ }^{\star} \text { in the } \\
\text { Christchurch cohort } \\
n=946\end{array}$ & $\begin{array}{c}\text { No. }(\%) \text { of } \\
\text { participants }{ }^{\star} \text { in the } \\
\text { Hamburg cohort } \\
n=1412\end{array}$ \\
\hline Age, mean $\pm S D$; yr & $66.5 \pm 16.6$ & $55.2 \pm 15.2$ & $64.5 \pm 12.8$ & $62.7 \pm 15.6$ \\
\hline Male sex & $515(47.2)$ & $476(59.9)$ & $569(60.1)$ & $899(63.7)$ \\
\hline Family history of coronary artery disease & $596(54.6)$ & $367(46.2)$ & $538(56.9)$ & $257(18.2)$ \\
\hline Hypertension & $767(70.2)$ & $397(49.9)$ & $566(59.8)$ & N/A \\
\hline Hypercholesterolemia & $646(59.2)$ & $381(47.9)$ & $542(57.3)$ & $\mathrm{N} / \mathrm{A}$ \\
\hline Diabetes mellitus & $319(29.2)$ & $104(13.1)$ & $165(17.4)$ & $193(13.7)$ \\
\hline Current smoking & $278(25.5)$ & $217(27.3)$ & $135(14.3)$ & $338(23.9)$ \\
\hline History of MI & $388(35.5)$ & $137(17.2)$ & $277(29.3)$ & $228(16.1)$ \\
\hline History of congestive heart failure & $211(19.3)$ & $39(4.9)$ & $80(8.5)$ & $211(14.9)$ \\
\hline History of angina & $288(26.4)$ & $184(23.1)$ & $421(44.5)$ & $\mathrm{N} / \mathrm{A}$ \\
\hline Detectable hs-cTnl concentration & $941(86.2)$ & $708(88.7)$ & $888(93.9)$ & $1185(83.9)$ \\
\hline Detectable hs-cTnT concentration & $922(84.4)$ & $573(72.1)$ & $680(71.9)$ & $899(63.7)$ \\
\hline 30-d MI or death $\dagger$ & $177(16.2)$ & $47(5.9)$ & $225(23.8)$ & $276(19.5)$ \\
\hline
\end{tabular}

(95\% Cl 3.31-6.47) with CCS hs-cTnT and 7.46 (95\% Cl 5.38-10.36) with CCS hs-cTnl.

The analytical performances of the hs-cTn assays in the 4 cohorts were in agreement with laboratory recommendations for hs-cTn assays (i.e., > 50\% above the limit of detection); ${ }^{6}$ the range of detectable hs-cTnl concentrations was $84 \%-94 \%$ and that for hs-cTnT concentrations was $64 \%-84 \%$ (Table 1 ). And across all 4 cohorts, the higher the CCS, the older the participant's age, the higher the hs-cTn level, the higher the glucose level and the lower the eGFR (Table 2). The prevalence of the primary outcome varied between the cohorts and hs-cTn assays, with about $50 \%-90 \%$ of participants with a CCS of 5 points having the outcome. However, among all participants in the 4 cohorts with normal laboratory results and a CCS of 0 , there was only 1 participant (male) who had testing with both hs-cTn assays and who had the primary outcome. The AUCs for CCS were higher in the Brisbane (hs-cTnI CCS AUC 0.95, 95\% Cl 0.930.97; hs-cTnT CCS AUC 0.91, 95\% Cl 0.87-0.94) and Christchurch (hs-cTnl CCS AUC 0.94, 95\% Cl 0.92-0.95; hs-cTnT CCS AUC 0.90, $95 \% \mathrm{Cl} 0.87-0.92$ ) cohorts compared with the Hamilton (hs-cTnl CCS AUC 0.87, 95\% Cl 0.85-0.89; hs-cTnT CCS AUC 0.83, 95\% Cl $0.81-0.85$ ) and Hamburg (hs-cTnI CCS AUC $0.86,95 \% \mathrm{Cl} 0.85-$ 0.89 ; hs-cTnT CCS AUC $0.84,95 \%$ Cl 0.81-0.86) cohorts (Supplemental Figure 3, Appendix 1).

In the Hamilton cohort, the sensitivity for a CCS of 0 points was 100\% (95\% Cl 97.9\%-100\%) with hs-cTnl (Table 3) and with hs-cTnT (Table 4). A CCS of 1 point yielded a sensitivity close to $99 \%$ with both hs-cTn assays. The specificity of a CCS of 5 points was higher for hs-cTnI CCS (95.6\%, 95\% CI 94.1\%-96.9\%) than for hs-cTnT CCS $(90.4 \%, 95 \%$ Cl 88.3\%-92.2\%).
The sensitivity of a CCS of 0 points in the Brisbane, Christchurch and Hamburg cohorts with hs-cTnl was $100 \%$ (Table 3). In each of these cohorts, the point-estimate specificity was greater than $95 \%$ with an hs-cTnl CCS of 5 points. For a hs-CTnT CCS of 0 points, the point-estimate sensitivity was greater than $99.5 \%$; the point-estimate specificity of a CCS of 5 points with the hs-cTnT assay was greater than $93.9 \%$ across the 3 non-North American cohorts (Table 4). A CCS of 1 point did not consistently achieve sensitivities greater than $99 \%$, with the hs-cTnT CCS of 1 yielding sensitivities of $98 \%$ or less in the 3 non-North American cohorts.

Combining all 4 cohort populations ( $n=4245$ participants), a CCS of 0 points yielded a sensitivity of $100 \%(95 \% \mathrm{Cl} 99.5 \%-$ $100 \%$ ) with no false negatives with hs-cTnl, and a sensitivity of 99.9\% (95\% Cl 99.2\%-100\%) with 1 false negative with hs-cTnT (Table 5). Because the sensitivity estimates are greater than 99.0\%, 378 participants (8.9\% of the population) would be classified as low risk using hs-cTnl with CCS and 444 participants (10.5\% of the population) using hs-cTnT with CCS. Applying a cut-off value for hs-cTnl of less than $5 \mathrm{ng} / \mathrm{L}$ alone, the sensitivity was $96.6 \%(95 \% \mathrm{Cl} 95.0 \%-97.8 \%)$ with 25 false negatives, and for hs-cTnT of less than $6 \mathrm{ng} / \mathrm{L}$ alone, the sensitivity was $98.2 \%$ (95\% Cl 97.0\%-99.0\%) with 13 false negatives. The specificity of a CCS of 5 points was $96.6 \%(95 \% \mathrm{Cl} 96.0 \%-$ $97.2 \%)$ with a PPV of $75.1 \%(95 \% \mathrm{Cl} 71.3 \%-78.5 \%)$ for hs-cTnl, and a specificity of $94.0 \%(95 \% \mathrm{Cl} 93.1 \%-94.7 \%)$ with a PPV of $61.7 \%$ (95\% Cl 58.1\%-65.2\%) for hs-cTnT. Applying the overall 99th percentile as the cut-off, the specificity was lower for hscTnl (> $26 \mathrm{ng} / \mathrm{L})$ at $93.2 \%(95 \% \mathrm{Cl}$ 92.3-94.0) and for hs-cTnT $(>14 \mathrm{ng} / \mathrm{L})$ at $73.8 \%(95 \% \mathrm{Cl} 72.3-75.2)$ compared with the respective CCSs of 5 . 
Assessing the performance of the CCS with regard to sex, in women, the specificity for the primary outcome was higher with a CCS of 5 (hs-CTnl CCS specificity $96.4 \%, 95 \%$ Cl 95.397.3; hs-cTnT CCS specificity $94.4 \%, 95 \% \mathrm{Cl} 93.1-95.5)$ compared with using the overall 99th percentile hs-cTn cut-offs (hs-cTnl specificity $93.6 \%, 95 \% \mathrm{Cl}$ 92.3-94.8; hs-cTnT specificity $76.1 \%, 95 \% \mathrm{Cl} 73.9-78.2$ ). Higher specificity with a CCS of
5 was evident in men as well (Supplemental Table 2, Appendix 3). Overall, there were no obvious differences between men and women in the performance of the CCS for the primary outcome.

For the secondary outcomes, the CCS algorithms also outperformed testing for hs-cTn alone for the index MI diagnosis (i.e., higher sensitivity and higher specificity; Supplemental

Table 2 (part 1 of 2): Participant characteristics and partitioning per the clinical chemistry score (range 0 to 5 points) at presentation to the emergency department for all cohorts, by tests for cardiac troponin I or $\mathrm{T}^{*}$

\begin{tabular}{|c|c|c|c|c|c|c|}
\hline Characteristic & Score 0 & Score 1 & Score 2 & Score 3 & Score 4 & Score 5 \\
\hline \multicolumn{7}{|l|}{ Hamilton cohort } \\
\hline \multicolumn{7}{|l|}{ hs-cTnl test } \\
\hline No. of participants & 91 & 208 & 264 & 264 & 146 & 119 \\
\hline Age, median (IQR); yr & $44(33-53)$ & $55(46-63)$ & $69(58-80)$ & $76(64-83)$ & $78(64-86)$ & $78(70-86)$ \\
\hline No. (\%) of male participants & $43(47.3)$ & $109(52.4)$ & $117(44.3)$ & $115(43.6)$ & $70(4847.9)$ & $61(51.3)$ \\
\hline hs-cTnl, median (IQR); ng/L & $1(<1-2)$ & $2(1-3)$ & $3(2-6)$ & $8(5-12)$ & $26(19-48)$ & $67(40-169)$ \\
\hline Glucose, median (IQR); mmol/L & $5.1(4.9-5.3)$ & $5.8(5.2-6.6)$ & $6.1(5.5-7.2)$ & $6.7(5.8-8.7)$ & $6.4(5.6-8.2)$ & $8.2(6.7-11.4)$ \\
\hline eGFR, median (IQR); mL/min/1.73 m² & $101(96-112)$ & $94(86-103)$ & $77(61-86)$ & $68(46-80)$ & $58(34-77)$ & $47(30-66)$ \\
\hline $\begin{array}{l}\text { No. (\%) of participants with MI or who died } \\
\text { within } 30 \mathrm{~d}\end{array}$ & $0(0)$ & $2(1.0)$ & $10(3.8)$ & $35(13.3)$ & $51(34.9)$ & $79(66.4)$ \\
\hline \multicolumn{7}{|l|}{ hs-cTnT test } \\
\hline No. of participants & 88 & 193 & 229 & 228 & 182 & 172 \\
\hline Age, median (IQR); yr & $44(34-54)$ & $54(45-62)$ & $64(56-76)$ & $75(63-83)$ & $80(68-86)$ & $79(70-86)$ \\
\hline No. (\%) of male participants & $41(46.6)$ & $101(52.3)$ & $101(44.1)$ & $114(50.0)$ & $83(45.6)$ & $95(55.2)$ \\
\hline hs-cTnT, median (IQR); ng/L & $4(<3-5)$ & $5(<3-7)$ & $8(5-12)$ & $13(10-16)$ & $28(23-49)$ & $56(39-96)$ \\
\hline Glucose, median (IQR); mmol/L & $5.1(4.9-5.3)$ & $5.7(5.1-6.6)$ & $6.1(5.5-7.8)$ & $6.8(5.8-8.4)$ & $5.9(5.4-7.7)$ & $7.7(5.6-10.3)$ \\
\hline eGFR, median (IQR); mL/min/1.73 m² & $101(96-110)$ & $94(87-103)$ & $81(69-89)$ & $69(53-81)$ & $60(40-77)$ & $44(27-65)$ \\
\hline $\begin{array}{l}\text { No. (\%) of participants with MI or who died } \\
\text { within } 30 \mathrm{~d}\end{array}$ & $0(0)$ & $2(1.0)$ & $10(4.4)$ & $29(12.7)$ & $52(28.6)$ & $84(48.8)$ \\
\hline \multicolumn{7}{|l|}{ Brisbane cohort } \\
\hline \multicolumn{7}{|l|}{ hs-cTnl test } \\
\hline No. of participants & 147 & 260 & 175 & 127 & 45 & 41 \\
\hline Age, median (IQR); yr & $46(38-52)$ & $50(41-59)$ & $57(47-66)$ & $64(54-77)$ & $72(55-82)$ & $76(65-83)$ \\
\hline No. (\%) of male participants & $77(52.4)$ & $158(60.8)$ & $111(63.4)$ & $81(63.8)$ & $25(55.6)$ & $24(58.5)$ \\
\hline hs-cTnl, median (IQR); ng/L & $2(2-2)$ & $2(2-3)$ & $4(3-5)$ & $6(4-12)$ & $30(18-83)$ & $130(52-273)$ \\
\hline Glucose, median (IQR); mmol/L & $5.2(4.9-5.3)$ & $5.7(5.2-6.4)$ & $6.1(5.6-7.2)$ & $6.4(5.7-8.0)$ & $6.3(5.6-8.3)$ & $8.0(6.6-9.2)$ \\
\hline eGFR, median (IQR); mL/min/1.73 m² & $104(97-112)$ & $99(91-107)$ & $84(72-95)$ & $74(57-86)$ & $70(42-70)$ & $60(42-74)$ \\
\hline $\begin{array}{l}\text { No. (\%) of participants with MI or who died } \\
\text { within } 30 \mathrm{~d}\end{array}$ & $0(0)$ & $0(0)$ & $1(0.6)$ & $9(7.1)$ & $13(28.9)$ & $26(63.4)$ \\
\hline \multicolumn{7}{|l|}{ hs-cTnT test } \\
\hline No. of participants & 162 & 250 & 176 & 108 & 53 & 46 \\
\hline Age, median (IQR); yr & $46(38-52)$ & $49(40-57)$ & $58(49-66)$ & $65(57-74)$ & $76(61-80)$ & $79(67-84)$ \\
\hline No. $(\%)$ of male participants & $84(51.9)$ & $161(64.4)$ & $106(60.2)$ & $69(63.9)$ & $28(52.8)$ & $28(60.9)$ \\
\hline hs-cTnT, median (IQR); ng/L & $4(3-5)$ & $5(4-7)$ & $8(6-11)$ & $12(9-17)$ & $28(22-40)$ & $69(45-134)$ \\
\hline Glucose, median (IQR); mmol/L & $5.1(4.9-5.3)$ & $5.8(5.3-6.4)$ & $6.1(5.4-7.1)$ & $6.6(5.9-8.0)$ & $6.7(5.6-9.0)$ & $7.6(6.5-8.5)$ \\
\hline eGFR, median (IQR); mL/min/1.73 m² & $104(97-112)$ & $100(92-108)$ & $84(73-92)$ & $73(61-84)$ & $64(43-78)$ & $55(36-72)$ \\
\hline $\begin{array}{l}\text { No. (\%) of participants with MI or who died } \\
\text { within } 30 \mathrm{~d}\end{array}$ & $0(0)$ & $1(0.4)$ & $5(2.8)$ & $9(8.3)$ & $9(17.0)$ & $25(54.4)$ \\
\hline
\end{tabular}


Table 2 (part 2 of 2): Participant characteristics and partitioning per the clinical chemistry score (range 0 to 5 points) at presentation to the emergency department for all cohorts, by tests for cardiac troponin I or $\mathrm{T}^{*}$

\begin{tabular}{|c|c|c|c|c|c|c|}
\hline Characteristic & Score 0 & Score 1 & Score 2 & Score 3 & Score 4 & Score 5 \\
\hline \multicolumn{7}{|l|}{ Christchurch cohort } \\
\hline \multicolumn{7}{|l|}{ hs-cTnl test } \\
\hline No. of participants & 32 & 154 & 266 & 220 & 132 & 142 \\
\hline Age, median (IQR); yr & $50(42-55)$ & $56(50-63)$ & $62(53-70)$ & $70(60-79)$ & $71(60-78)$ & $71(62-80)$ \\
\hline No. (\%) of male participants & $25(78.1)$ & $88(57.1)$ & $137(51.5)$ & $134(60.9)$ & $87(65.9)$ & $98(69.0)$ \\
\hline hs-cTnl median ng/L (IQR) & $2(2-3)$ & $2(2-3)$ & $3(2-5)$ & $7(5-11)$ & $28(20-134)$ & $231(75-1099)$ \\
\hline Glucose median mmol/L (IQR) & $5.1(5-5.4)$ & $5.3(5.1-5.7)$ & $5.9(5.3-6.7)$ & $6.5(5.8-7.6)$ & $6.2(5.5-7.6)$ & $7.1(6.0-9.1)$ \\
\hline eGFR median $\mathrm{mL} / \mathrm{min} / 1.73 \mathrm{~m}^{2}$ (IQR) & $98(93-105)$ & $85(76-95)$ & $74(64-81)$ & $67(56-80)$ & $64(49-79)$ & $63(48-74)$ \\
\hline $\begin{array}{l}\text { No. (\%) of participants with MI or who died } \\
\text { within } 30 \mathrm{~d}\end{array}$ & $0(0)$ & $1(0.6)$ & $2(0.8)$ & $27(12.3)$ & $68(51.5)$ & $127(89.4)$ \\
\hline \multicolumn{7}{|l|}{ hs-cTnT test } \\
\hline No. of participants & 43 & 191 & 269 & 168 & 129 & 146 \\
\hline Age, median (IQR); yr & $50(45-58)$ & $57(50-63)$ & $61(54-69)$ & $73(64-79)$ & $73(61-80)$ & $73(64-80)$ \\
\hline No. $(\%)$ of male participants & $29(67.4)$ & $114(59.7)$ & $135(50.2)$ & $104(61.9)$ & $85(65.9)$ & $102(69.9)$ \\
\hline hs-cTnT, median (IQR); ng/L & $3(3-4)$ & $4(3-6)$ & $5(3-8)$ & $13(10-16)$ & $30(22-49)$ & $85(47-223)$ \\
\hline Glucose, median (IQR); mmol/L & $5.1(4.9-5.3)$ & $5.3(5.1-5.7)$ & $6.1(5.6-7.1)$ & $6.6(5.9-7.8)$ & $6.0(5.4-7.4)$ & $7.0(6.0-8.9)$ \\
\hline eGFR, median (IQR); mL/min/1.73 m² & $97(93-104)$ & $83(75-93)$ & $75(67-82)$ & $64(56-73)$ & $65(48-81)$ & $60(44-71)$ \\
\hline $\begin{array}{l}\text { No. (\%) of participants with MI or who died } \\
\text { within } 30 \mathrm{~d}\end{array}$ & $0(0)$ & $6(3.1)$ & $8(3.0)$ & $24(14.3)$ & $75(58.1)$ & $112(76.7)$ \\
\hline \multicolumn{7}{|l|}{ Hamburg cohort } \\
\hline \multicolumn{7}{|l|}{ hs-cTnl test } \\
\hline No. of participants & 108 & 225 & 347 & 404 & 153 & 175 \\
\hline Age, median (IQR); yr & $43(33-52)$ & $52(43-62)$ & $62(50-73)$ & $71(61-77)$ & $72(60-79)$ & $73(64-79)$ \\
\hline No. $(\%)$ of male participants & $60(55.6)$ & $142(63.1)$ & $211(60.8)$ & $277(68.6)$ & $104(68.0)$ & $105(60.0)$ \\
\hline hs-cTnl, median (IQR); ng/L & $2(2-3)$ & $2(2-4)$ & $4(2-8)$ & $8(5-12)$ & $27(19-114)$ & $172(59-749)$ \\
\hline Glucose, median (IQR); mmol/L & $5.1(4.8-5.3)$ & $5.4(5.0-5.8)$ & $5.8(5.3-6.7)$ & $6.5(5.9-7.9)$ & $6.1(5.6-7.4)$ & $7.1(6.1-9.6)$ \\
\hline eGFR, median (IQR); mL/min/1.73 m² & $103(97-112)$ & $94(86-105)$ & $80(66-91)$ & $67(53-82)$ & $60(45-80)$ & $58(39-72)$ \\
\hline $\begin{array}{l}\text { No. (\%) of participants with MI or who died } \\
\text { within } 30 \mathrm{~d}\end{array}$ & $0(0)$ & $2(0.9)$ & $20(5.8)$ & $60(14.9)$ & $68(44.4)$ & $126(72.0)$ \\
\hline \multicolumn{7}{|l|}{ hs-cTnT test } \\
\hline No. of participants & 151 & 254 & 338 & 311 & 168 & 190 \\
\hline Age, median (IQR); yr & $43(34-54)$ & $52(45-61)$ & $62(51-72)$ & $73(64-78)$ & $73(62-79)$ & $75(66-82)$ \\
\hline No. (\%) of male participants & $86(57.0)$ & $160(63.0)$ & $207(61.2)$ & $209(67.2)$ & $120(71.4)$ & $117(61.6)$ \\
\hline hs-cTnT, median (IQR); ng/L & $4(<3-5)$ & $5(<3-6)$ & $7(5-10)$ & $13(10-17)$ & $28(23-56)$ & $74(41-188)$ \\
\hline Glucose, median (IQR); mmol/L & $5.1(4.8-5.3)$ & $5.6(5.2-6.0)$ & $6.0(5.5-6.9)$ & $6.5(5.8-8.0)$ & $6.2(5.6-7.7)$ & $7.2(6.2-9.7)$ \\
\hline eGFR, median (IQR); mL/min/1.73 m² & $103(96-112)$ & $94(82-102)$ & $78(65-87)$ & $67(53-80)$ & $57(43-77)$ & $51(35-69)$ \\
\hline $\begin{array}{l}\text { No. (\%) of participants with MI or who died } \\
\text { within } 30 \mathrm{~d}\end{array}$ & $1(0.7)$ & $10(3.9)$ & $25(7.4)$ & $49(15.8)$ & $70(41.7)$ & $121(63.7)$ \\
\hline
\end{tabular}

Note: eGFR = estimated glomerular filtration rate, hs-cTnI = high-sensitivity cardiac troponin I, hs-cTnT = high-sensitivity cardiac troponin T, IQR = interquartile range, MI = myocardial infarction.

*Values rounded to nearest whole number except for glucose.

Table 3, Appendix 3). With respect to interventions, there was nearly a doubling per score in the revascularization rate, with a hs-cTnl CCS of 0 yielding $0.79 \%$ ( $95 \% \mathrm{Cl} 0.16-2.32)$ compared with a hs-cTnl CCS of 5 with $26.2 \%(95 \% \mathrm{Cl} 21.8-31.2)$ revascu- larization rate $(p>0.05$ between hs-cTnl CCS and hs-cTnT CCS; Table 6). The sensitivities of the CCS for 30 -day unstable angina, $\mathrm{MI}$ and death (sensitivity for a hs-cTnI CCS of 0 points 99.3\%, 95\% Cl 98.3-99.8; for a hs-cTnT CCS of 0 points $98.8 \%$, 
Table 3: Diagnostic accuracy of the clinical chemistry score with high-sensitivity cardiac troponin I for myocardial infarction or death at 30 days after presentation to the emergency department, by cohort

\begin{tabular}{|c|c|c|c|c|c|c|}
\hline Score & $\begin{array}{c}\text { Sensitivity, \% } \\
(95 \% \mathrm{CI})\end{array}$ & $\begin{array}{l}\text { Specificity, \% } \\
\text { (95\% CI) }\end{array}$ & $\begin{array}{l}\text { Negative predictive } \\
\text { value, } \% \\
(95 \% \mathrm{Cl})\end{array}$ & $\begin{array}{c}\text { Positive predictive } \\
\text { value, } \% \\
(95 \% \mathrm{CI})\end{array}$ & $\begin{array}{c}\text { Negative } \\
\text { likelihood ratio } \\
(95 \% \mathrm{CI})\end{array}$ & $\begin{array}{c}\text { Positive } \\
\text { likelihood ratio } \\
(95 \% \mathrm{CI})\end{array}$ \\
\hline \multicolumn{7}{|c|}{ Hamilton cohort, $n=1092$} \\
\hline 0 & $100(97.9-100)$ & $10.0(8.1-12.1)$ & $100(95.1-100)$ & $17.7(17.4-18)$ & $0(0-0.21)$ & $1.11(1.08-1.13)$ \\
\hline 1 & $98.9(96.0-99.8)$ & $32.5(29.4-35.6)$ & $99.3(97.4-99.8)$ & $22.1(21.3-22.9)$ & $0.03(0.01-0.12)$ & $1.46(1.40-1.54)$ \\
\hline 5 & $44.6(37.1-52.3)$ & $95.6(94.1-96.9)$ & $89.9(88.6-91.1)$ & $66.4(58.3-73.6)$ & $0.58(0.50-0.65)$ & $10.2(7.2-14.4)$ \\
\hline \multicolumn{7}{|c|}{ Brisbane cohort, $n=795$} \\
\hline 0 & $100(92.7-100)$ & $19.7(16.9-22.7)$ & $100(97.5-100)$ & $7.6(5.6-9.9$ & $0(0-0.37)$ & $1.25(1.13-1.29)$ \\
\hline 1 & $100(92.7-100)$ & $54.6(50-9-58.2)$ & $100(99.1-100)$ & $12.6(9.5-16.4)$ & $0(0-0.13)$ & $2.20(1.97-2.37)$ \\
\hline 5 & $53.1(38.3-67.5)$ & $98.0(96.7-98.9)$ & $96.9(95.5-98.1)$ & $63.4(46.9-77.9)$ & $0.48(0.43-0.61)$ & $26.4(15.0-45.9)$ \\
\hline \multicolumn{7}{|c|}{ Christchurch cohort, $n=946$} \\
\hline 0 & $100(97.6-100)$ & $4.4(3.1-6.2)$ & $100(84.2-100)$ & $24.6(21.9-27.5)$ & $0(0-0.38)$ & $1.05(1.02-1.06)$ \\
\hline 1 & $99.6(97.5-100)$ & $25.7(22.5-29.0)$ & $99.5(97-100)$ & $29.5(26.3-32.9)$ & $0.02(0-0.10)$ & $1.34(1.28-1.40)$ \\
\hline 5 & $56.4(49.7-63.0)$ & $97.9(96.6-98.8)$ & $87.8(85.3-90.0)$ & $89.4(83.2-94.0)$ & $0.44(0.38-0.51)$ & $27.1(16.4-45.2)$ \\
\hline \multicolumn{7}{|c|}{ Hamburg cohort, $n=1412$} \\
\hline 0 & $100(98.6-100)$ & $9.5(7.9-11.4)$ & $100(96.6-100)$ & $21.2(19.0-23.5)$ & $0(0-0.14)$ & $1.11(1.08-1.13)$ \\
\hline 1 & $99.3(97.4-99.8)$ & $29.1(26.6-31.8)$ & $99.4(97.8-99.8)$ & $25.4(22.9-28.1)$ & $0.02(0.01-0.09)$ & $1.40(1.35-1.46)$ \\
\hline 5 & $45.7(39.9-51.5)$ & $95.7(94.3-96.7)$ & $87.9(85.9-89.6)$ & $72.0(64.9-78.1)$ & $0.57(0.51-0.63)$ & $10.6(7.8-14.3)$ \\
\hline
\end{tabular}

Note: $\mathrm{Cl}$ = confidence interval.

Table 4: Diagnostic accuracy of the clinical chemistry score with high-sensitivity cardiac troponin $\mathrm{T}$ for myocardial infarction or death at 30 days after presentation to the emergency department, by cohort

\begin{tabular}{|c|c|c|c|c|c|c|}
\hline Score & $\begin{array}{c}\text { Sensitivity, \% } \\
(95 \% \mathrm{CI})\end{array}$ & $\begin{array}{c}\text { Specificity, \% } \\
(95 \% \mathrm{Cl})\end{array}$ & $\begin{array}{l}\text { Negative predictive } \\
\text { value, \% } \\
(95 \% \mathrm{CI})\end{array}$ & $\begin{array}{l}\text { Positive predictive } \\
\text { value, } \% \\
(95 \% \mathrm{Cl})\end{array}$ & $\begin{array}{l}\text { Negative } \\
\text { likelihood ratio } \\
(95 \% \mathrm{CI})\end{array}$ & $\begin{array}{c}\text { Positive } \\
\text { likelihood ratio } \\
(95 \% \mathrm{CI})\end{array}$ \\
\hline \multicolumn{7}{|c|}{ Hamilton cohort, $n=1092$} \\
\hline 0 & $100(97.9-100)$ & $9.6(7.8-11.7)$ & $100(95.0-100)$ & $17.6(17.3-17.9)$ & $0(0-0.22)$ & $1.11(1.07-1.13)$ \\
\hline 1 & $98.9(96.0-99.9)$ & $30.5(27.5-33.6)$ & $99.3(97.2-99.8)$ & $21.6(20.8-22.4)$ & $0.04(0.01-0.13)$ & $1.42(1.36-1.49)$ \\
\hline 5 & $47.5(39.9-55.1)$ & $90.4(88.3-92.2)$ & $89.9(88.5-91.1)$ & $48.8(24.6-55.1)$ & $0.58(0.50-0.66)$ & $4.9(3.8-6.3)$ \\
\hline \multicolumn{7}{|c|}{ Brisbane cohort, $n=795$} \\
\hline 0 & $100(92.7-100)$ & $21.7(18.8-24.8)$ & $100(97.7-100)$ & $7.7(5.8-10.1)$ & $0(0-0.34)$ & $1.28(1.16-1.32)$ \\
\hline 1 & $98(89.1-99.9)$ & $55.1(51.4-58.7)$ & $99.8(98.7-100)$ & $12.5(9.4-16.3)$ & $0.04(0.01-0.19)$ & $2.18(1.96-2.38)$ \\
\hline 5 & $51(36.3-65.6)$ & $97.2(95.7-98.2)$ & $96.8(95.3-97.9)$ & $54.3(39.0-69.1)$ & $0.50(0.37-0.64)$ & $18.1(10.9-29.6)$ \\
\hline \multicolumn{7}{|c|}{ Christchurch cohort, $n=946$} \\
\hline 0 & $100(97.6-100)$ & $6.0(4.3-7.9)$ & $100(88.0-100)$ & $24.9(22.1-27.9)$ & $0(0-0.28)$ & $1.06(1.04-1.08)$ \\
\hline 1 & $97.3(94.3-99.0)$ & $31.6(28.2-35.2)$ & $97.4(94.5-99.1)$ & $30.8(27.4-34.3)$ & $0.08(0.04-0.18)$ & $1.42(1.35-1.50)$ \\
\hline 5 & $49.8(43.1-56.5)$ & $95.3(93.5-96.7)$ & $85.9(83.3-88.2)$ & $76.7(69.0-83.3)$ & $0.53(0.46-0.60)$ & $10.6(7.4-15.0)$ \\
\hline \multicolumn{7}{|c|}{ Hamburg cohort, $n=1412$} \\
\hline 0 & $99.6(98.0-100)$ & $13.2(11.4-15.3)$ & $99.3(96.3-100)$ & $21.8(19.6-24.2)$ & $0.03(0-0.15)$ & $1.15(1.12-1.18)$ \\
\hline 1 & $96.0(93.0-97.8)$ & $34.7(32.0-37.5)$ & $97.3(95.2-98.5)$ & $26.3(23.7-29.1)$ & $0.11(0.06-0.20)$ & $1.47(1.40-1.54)$ \\
\hline 5 & $43.8(38.1-49.7)$ & $93.9(92.4-95.2)$ & $87.3(85.3-89.1)$ & $63.7(56.6-70.2)$ & $0.60(0.53-0.66)$ & $7.2(5.5-9.4)$ \\
\hline
\end{tabular}

Note: $\mathrm{Cl}=$ confidence interval. 
Table 5 (part 1 of 2): Performance of the clinical chemistry score for identifying patients at low and high risk for myocardial infarction or death within $\mathbf{3 0}$ days after presentation to the emergency department*

\begin{tabular}{|c|c|c|}
\hline Score cut-off & $\begin{array}{c}\text { No. of } \\
\text { participants } \\
\text { with MI or who } \\
\text { died } †\end{array}$ & $\begin{array}{c}\text { No. of } \\
\text { participants } \\
\text { with no MI or } \\
\text { death }\end{array}$ \\
\hline \multicolumn{3}{|l|}{ CCS with hs-cTnl test } \\
\hline $\mathrm{CCS}>0$ & 727 & 3140 \\
\hline $\mathrm{CCS}=0$ & 0 & 378 \\
\hline Sensitivity, \% (95\% Cl) & $100(99.5-100)$ & \\
\hline NPV, \% (95\% Cl) & $100(98.9-100)$ & \\
\hline Negative likelihood ratio & $0(0-0.05)$ & \\
\hline $\begin{array}{l}\text { Patients at low risk, \% (95\% } \\
\text { Cl) }\end{array}$ & $8.9(8.1-9.8)$ & \\
\hline $\mathrm{CCS}=5$ & 358 & 119 \\
\hline $\mathrm{CCS}<5$ & 369 & 3399 \\
\hline Specificity, \% (95\% Cl) & $96.6(96.0-97.2)$ & \\
\hline PPV, \% (95\% Cl) & $75.1(71.3-78.5)$ & \\
\hline Positive likelihood ratio & $14.6(12.0-17.6)$ & \\
\hline $\begin{array}{l}\text { Patients at high risk, } \% \text { (95\% } \\
\text { Cl) }\end{array}$ & $11.2(10.3-12.2)$ & \\
\hline \multicolumn{3}{|l|}{ CCS with hs-cTnT test } \\
\hline $\mathrm{CCS}>0$ & 726 & 3075 \\
\hline $\mathrm{CCS}=0$ & 1 & 443 \\
\hline Sensitivity, \% (95\% CI) & $99.9(99.2-100)$ & \\
\hline NPV, \% (95\% Cl) & $99.8(98.4-100)$ & \\
\hline Negative likelihood ratio & $0.01(0-0.06)$ & \\
\hline $\begin{array}{l}\text { Patients at low risk, } \%(95 \% \\
\text { Cl) }\end{array}$ & $10.5(9.6-11.4)$ & \\
\hline $\mathrm{CCS}=5$ & 342 & 212 \\
\hline $\mathrm{CCS}<5$ & 385 & 3306 \\
\hline Specificity, \% (95\% CI) & $94.0(93.1-94.7)$ & \\
\hline PPV, \% (95\% Cl) & $61.7(58.1-65.2)$ & \\
\hline Positive likelihood ratio & $7.8(6.7-9.1)$ & \\
\hline $\begin{array}{l}\text { Patients at high-risk, \% (95\% } \\
\mathrm{Cl})\end{array}$ & $13.1(12.1-14.1)$ & \\
\hline \multicolumn{3}{|l|}{ hs-cTnl test only } \\
\hline$\geq 5 \mathrm{ng} / \mathrm{L}$ & 702 & 1543 \\
\hline$<5 \mathrm{ng} / \mathrm{L}$ & 25 & 1975 \\
\hline Sensitivity, \% (95\% Cl) & $96.6(95.0-97.8)$ & \\
\hline NPV, \% (95\% Cl) & $98.8(98.2-99.2)$ & \\
\hline Negative likelihood ratio & $0.06(0.04-0.09)$ & \\
\hline$>26 \mathrm{ng} / \mathrm{L}$ & 537 & 239 \\
\hline$\leq 26 \mathrm{ng} / \mathrm{L}$ & 190 & 3279 \\
\hline Specificity, \% (95\% CI) & $93.2(92.3-94.0)$ & \\
\hline PPV, \% (95\% Cl) & $69.2(65.8-72.4)$ & \\
\hline Positive likelihood ratio & $10.9(9.6-12.4)$ & \\
\hline
\end{tabular}

Table 5 (part 2 of 2): Performance of the clinical chemistry score for identifying patients at low and high risk for myocardial infarction or death within $\mathbf{3 0}$ days after presentation to the emergency department*

\begin{tabular}{|c|c|c|}
\hline Score cut-off & $\begin{array}{l}\text { No. of } \\
\text { participants } \\
\text { with MI or who } \\
\text { diedt }\end{array}$ & $\begin{array}{l}\text { No. of } \\
\text { participants } \\
\text { with no MI or } \\
\text { death }\end{array}$ \\
\hline \multicolumn{3}{|l|}{ hs-cTnT test only } \\
\hline$\geq 6 \mathrm{ng} / \mathrm{L}$ & 714 & 2262 \\
\hline$<6 \mathrm{ng} / \mathrm{L}$ & 13 & 1256 \\
\hline Sensitivity, \% (95\% CI) & $98.2(97.0-99.0)$ & \\
\hline NPV, \% (95\% Cl) & $99.0(98.3-99.5)$ & \\
\hline Negative likelihood ratio & $0.05(0.03-0.09)$ & \\
\hline$>14 \mathrm{ng} / \mathrm{L}$ & 640 & 922 \\
\hline$\leq 14 \mathrm{ng} / \mathrm{L}$ & 87 & 2596 \\
\hline Specificity, \% (95\% Cl) & $73.8(72.3-75.2)$ & \\
\hline PPV, \% (95\% Cl) & $41.0(38.5-43.5)$ & \\
\hline Positive likelihood ratio & $3.4(3.2-3.6)$ & \\
\hline \multicolumn{3}{|c|}{$\begin{array}{l}\text { Note: } \mathrm{CCS}=\text { clinical chemistry score, } \mathrm{Cl}=\text { confidence interval, hs-cTnl = high-sensitivity } \\
\text { cardiac troponin I, hs-cTnT = high-sensitivity cardiac troponin T, MI = myocardial } \\
\text { infarction, NPV = negative predictive value, PPV = positive predictive value. } \\
{ }^{*} \text { Combined cohorts, } n=4245 \text {. } \\
\text { †Unless specified otherwise. }\end{array}$} \\
\hline
\end{tabular}

95\% Cl 97.6-99.5) were significantly higher than those for hscTn alone (sensitivity for hs-cTnl $<5 \mathrm{ng} / \mathrm{L} 87.7 \%, 95 \% \mathrm{Cl} 84.8-$ 90.2; for hs-cTnT $<6 \mathrm{ng} / \mathrm{L} 93.0 \%, 95 \% \mathrm{Cl}$ 90.6-94.9). The specificity of a CCS of 5 was also significantly higher than using the overall 99th percentiles of hs-cTn (Supplemental Table 4, Appendix 3).

\section{Interpretation}

The CCS coupled with other existing accelerated diagnostic pathways ${ }^{1,30,34}$ may further identify patients at low and high risk of MI or death on presentation to the emergency department.

The goal for electrocardiograms, testing for laboratory biomarkers and clinical algorithms when patients present to the emergency department with symptoms suggestive of acute coronary syndrome is both diagnostic and for risk stratification. For identifying low-risk patients who may be suitable for discharge, many different approaches have been proposed, with the simplest being a low or undetectable hs-cTn concentration. ${ }^{2,3}$ Despite the ease of these approaches, using hs-cTn alone has not reliably achieved a sensitivity greater than $99.0 \%$ to safely rule out MI and discharge patients presenting to the emergency department in large populations. ${ }^{2,3}$ At the other extreme, for rule in of $\mathrm{MI}$ and identifying patients at high risk who require hospital admission, besides the use of electrocardiograms, algorithms that have used hs-cTn tests alone use cut-offs that are several-fold higher than the published 99th percentiles. ${ }^{35}$ The required specificity or PPV for algorithms to identify patients at high risk have not undergone the same methodological approach as the sensitivity estimate..$^{30}$ 
Table 6: Rate of revascularization* per clinical chemistry score in participants at 30 days after presentation to the emergency department in the overall cohort populationt

\section{Intervention rate by score \\ of the CCS}

\section{CCS with hs-cTnl test}

Score and no. of participants

No. of events

$\%(95 \% \mathrm{Cl})$

\section{CCS with hs-cTnT test}

Score and no. of participants

No. of events

$\%(95 \% \mathrm{Cl})$

$p$ value between rates for

hs-cTnl and hs-cTnT

\section{Rate of revascularization}

$$
\begin{array}{cccccc}
\operatorname{CCS} 0 & \operatorname{CCS} 1 & \operatorname{cCS} 2 & \operatorname{cCS} 3 & \operatorname{ccs} 4 & \operatorname{cCS} 5 \\
n=378 & n=847 & n=1052 & n=1015 & n=476 & n=477
\end{array}
$$

13

38

75

67

125

$0.79(0.16-2.32) \quad 1.54(0.82-2.63) \quad 3.61(2.56-4.96)$

$7.39(5.81-9.26)$

$14.1(10.9-17.9)$

$26.2(21.8-31.2)$

$$
\begin{gathered}
\operatorname{CCS} 0 \\
n=444
\end{gathered}
$$

6

$1.35(0.50-2.94)$

0.5

\begin{abstract}
CCS 1
\end{abstract}
19

$$
\begin{gathered}
\operatorname{CCS} 2 \\
n=1012
\end{gathered}
$$

43

$2.14(1.29-3.34)$
$4.25(3.08-5.72)$

0.9
CCS 3
$n=815$

65

\section{CCS 4}

$n=532$

73

13.7 (10.8-17.3)

0.9
CCS 5

$n=554$

115

20.8 (17.1-24.9)

0.1

Note: $\mathrm{CCS}=$ clinical chemistry score, $\mathrm{Cl}$ = confidence interval, hs-cTnI = high-sensitivity cardiac troponin I, hs-cTnT = high-sensitivity cardiac troponin T.

*Percutaneous coronary intervention or coronary artery bypass graft.

tCombined cohorts, $n=4245$.

Our proposed CCS includes measurements and cut-offs for accepted normality for glucose and eGFR, and includes measurable normal concentrations of hs-cTn, all of which permits clinical laboratories to monitor these cut-offs with quality control procedures to ensure reliable and accurate results. These are essential elements for the provision of optimal laboratory services. Algorithms that use the lower limit of reporting, such as the limit of detection, for hs-cTn assays are approaches that pose a safety risk for patients, because this metric is not monitored by clinical laboratories and is subject to analytical bias, with wide variation on repeat measurements on different instruments and different lots of reagents; all of which may lead to patient misclassification. ${ }^{4,6-8,36}$ Just as important is that there is no standardization on reporting at the low end: in a 2018 quality survey, undetectable ranges were reported from clinical laboratories that ranged from less than 1 to less than $10 \mathrm{ng} / \mathrm{L}$ for the Abbott hs-cTnl assay and less than 3 to less than $13 \mathrm{ng} / \mathrm{L}$ for the Roche hs-cTnT assay. ${ }^{37}$ In this regard, physicians using "undetectable concentrations" will misclassify patients depending on how the clinical laboratory reports their results, if they use results from hs-cTn testing alone.

We based the inclusion of different concentration ranges for different hs-cTn scores on published low-risk cutoffs, clinical outcomes, observed analytical variation and different 99th percentiles. ${ }^{19,26-32}$ Importantly, these concentrations can be monitored by laboratories via different quality assurance procedures, which will mitigate misclassification of patients owing to unacceptable analytical variation. ${ }^{5,38}$ The CCS was reproducible across different sample types (ethylenediaminetetraacetic acid plasma, lithium heparin plasma and serum), and under different storage and preanalytical conditions, which should be reassuring to physicians and clinical laboratories, because hs-cTn measurements alone can be affected by different blood collection tubes and preana- lytical interferences. ${ }^{6,39}$ Some of these preanalytical sources of variation easily exceed the analytical imprecision benchmark of $10 \%$ for hs-cTn assays, and despite attempts to sensitize the clinical community to the importance of this matter, it still represents a substantial gap in optimizing patient care. ${ }^{6,39}$

Adoption of the CCS algorithm would standardize reporting of hs-cTn test results, how the tests are interpreted in the normal range, and represent an option less susceptible to both analytical and preanalytical errors. This could result in the safest laboratory approach for physicians to use at presentation in the emergency department. Specifically, for physicians who do not want to miss an outcome or patients who want reassurance that they are at low risk after the first blood draw, a CCS of 0 would safely classify close to $10 \%$ of the population in the emergency department who present with symptoms of acute coronary syndrome as being at low risk for unstable angina, $\mathrm{MI}$, revascularization procedures and death. Those with a CCS of 5 points would be at high risk, with the remaining patients needing additional serial testing of hs-cTn using the $0 / 1$ hour, $0 / 2$ hour or $0 / 3$ hour blood sampling protocols. ${ }^{30,34}$

\section{Limitations}

First, only about $10 \%$ of patients would be considered to be at low risk; this is similar to other studies rendering results for hs-cTn tests as detectable versus nondetectable. ${ }^{3}$ However, as hs-cTn assays become even more analytically sensitive and precise at lower concentrations, it will be expected that all individuals will have a detectable concentration of cardiac troponin, which would make this cut-off obsolete. Second, we elected to assess outcomes only to 30 days after presentation to the emergency department. A longer-term outcome assessment may be beneficial for some clinicians, but an acute outcome within 30 days of presentation to the emergency department might be sufficient for those physicians 
involved in the setting of acute care. Third, despite our intention to use MI as a common outcome across the cohorts, we emphasize that each site employed different cardiac troponin assays and change criteria (i.e., rise or fall of cardiac troponin levels) for the adjudication of MI. Only a prospective study using the same cardiac troponin assay, the same rise/fall change criteria in cardiac troponin levels, and adjudication criteria would ensure that the patients were assessed in a similar manner across the different sites.

\section{Conclusion}

A CCS of 0 points, indicating that all laboratory test levels are normal, can achieve the clinically recommended sensitivity of greater than $99.0 \%$ for 30-day MI and death, and can identify about $10 \%$ of patients as at low risk and suitable for discharge in the acute care setting. A CCS of 5 points, indicating that all laboratory test levels are abnormal, can identify patients at high risk who are suitable for hospital admission and evaluation, with about $10 \%$ of patients identified as being at high risk. The similar performance of the CCS using either hs-cTnl or hs-cTnT in different geographical locations and emergency department populations calls for a clinical study to evaluate its clinical effectiveness prospectively.

\section{References}

1. Roffi M, Patrono C, Collet JP, et al.; ESC Scientific Document Group. 2015 ESC Guidelines for the management of acute coronary syndromes in patients presenting without persistent ST-segment elevation: Task Force for the Management of Acute Coronary Syndromes in Patients Presenting without Persistent ST-Segment Elevation of the European Society of Cardiology (ESC). Eur Heart J 2016;37:267-315

2. Pickering JW, Than MP, Cullen L, et al. Rapid rule-out of acute myocardial infarction with a single high-sensitivity cardiac troponin T measurement below the limit of detection: a collaborative meta-analysis. Ann Intern Med 2017; 166:715-24.

3. Chapman AR, Lee KK, McAllister DA, et al. Association of high-sensitivity cardiac troponin I concentration with cardiac outcomes in patients with suspected acute coronary syndrome. JAMA 2017;318:1913-24.

4. Lyon AW, Kavsak PA, Lyon OA, et al. Simulation models of misclassification error for single thresholds of high-sensitivity cardiac troponin I due to assay bias and imprecision. Clin Chem 2017;63:585-92.

5. Kavsak PA, Jaffe AS, Greene DN, et al. Total analytic error for low cardiac troponin concentrations ( $\leq 10 \mathrm{ng} / \mathrm{L}$ ) by use of a high-sensitivity cardiac troponin assay. Clin Chem 2017;63:1043-5.

6. Wu AHB, Christenson R, Greene D, et al. Clinical laboratory practice recommendations for the use of cardiac troponin in acute coronary syndrome: expert opinion from the Academy of the American Association for Clinical Chemistry and the Task Force on Clinical Applications of Cardiac Bio-Markers of the International Federation of Clinical Chemistry and Laboratory Medicine. Clin Chem 2018;64:645-55.

7. Apple FS, Jaffe AS. Clinical implications of a recent adjustment to the highsensitivity cardiac troponin T assay: user beware. Clin Chem 2012;58:1599-600.

8. Kavsak PA, Worster A, Oliver R, et al. Variability between reagent lots for highsensitivity cardiac troponin I may affect performance of early-rule out strategies. Can J Cardiol 2018;34:209.e5-6.

9. Kavsak PA, Worster A. Dichotomizing high-sensitivity cardiac troponin T results and important analytical considerations. J Am Coll Cardiol 2012;59:1570.

10. Herman DS, Kavsak PA, Greene DN. Variability and error in cardiac troponin testing: an ACLPS critical review. Am J Clin Pathol 2017;148:281-95.

11. Shortt C, Ma J, Clayton N, et al. Rule-in and rule-out of myocardial infarction using cardiac troponin and glycemic biomarkers in patients with symptoms suggestive of acute coronary syndrome. Clin Chem 2017;63:403-14.
12. Shortt C, Xie F, Whitlock R, et al. Economic considerations of early rule-in/rule-out algorithms for the diagnosis of myocardial infarction in the emergency department using cardiac troponin and glycemic biomarkers. Clin Chem 2017;63: 593-602.

13. Greenslade JH, Cullen L, Kalinowski L, et al. Examining renal impairment as a risk factor for acute coronary syndrome: a prospective observational study. Ann Emerg Med 2013;62:38-46.e1.

14. Timmer JR, Hoekstra M, Nijsten MW, et al. Prognostic value of admission glycosylated hemoglobin and glucose in nondiabetic patients with ST-segmentelevation myocardial infarction treated with percutaneous coronary intervention. Circulation 2011;124:704-11.

15. AlFaleh HF, Alsuwaida AO, Ullah A, et al. Glomerular filtration rate estimated by the CKD-EPI formula is a powerful predictor of in-hospital adverse clinical outcomes after an acute coronary syndrome. Angiology 2012;63:119-26.

16. Kavsak PA, Shortt C, Ma J, et al. A laboratory score at presentation to rule-out serious cardiac outcomes or death in patients presenting with symptoms suggestive of acute coronary syndrome. Clin Chim Acta 2017;469:69-74.

17. Shouval R, Hadanny A, Shlomo N, et al. Machine learning for prediction of 30-day mortality after ST elevation myocardial infraction: an Acute Coronary Syndrome Israeli Survey data mining study. Int J Cardiol 2017;246:7-13.

18. Than M, Herbert M, Flaws D, et al. What is an acceptable risk of major adverse cardiac event in chest pain patients soon after discharge from the emergency department?: A clinical survey. Int J Cardiol 2013;166:752-4.

19. Kavsak PA, Worster A, Ma J, et al. High-sensitivity cardiac troponin risk cutoffs for acute cardiac outcomes at emergency department presentation. Can J Cardiol 2017;33:898-903.

20. Than M, Cullen L, Aldous S, et al. 2-hour accelerated diagnostic protocol to assess patients with chest pain symptoms using contemporary troponins as the only biomarker: the ADAPT trial. J Am Coll Cardiol 2012;59:2091-8.

21. Than MP, Pickering JW, Aldous SJ, et al. Effectiveness of EDACS versus ADAPT accelerated diagnostic pathways for chest pain: a pragmatic randomized controlled trial embedded within practice. Ann Emerg Med 2016;68:93-102.e1.

22. Neumann JT, Sörensen NA, Schwemer T, et al. Diagnosis of myocardial infarction using a high-sensitivity troponin I 1-hour algorithm. JAMA Cardiol 2016; 1:397-404.

23. Neumann JT, Sörensen NA, Ojeda F, et al. Immediate rule-out of acute myocardial infarction using electrocardiogram and baseline high-sensitivity troponin I. Clin Chem 2017;63:394-402.

24. Thygesen K, Alpert JS, Jaffe AS, et al. Third universal definition of myocardial infarction. Circulation 2012;126:2020-35.

25. Greenslade $\mathrm{JH}$, Kavsak P, Parsonage W, et al. Combining presentation highsensitivity cardiac troponin I and glucose measurements to rule-out an acute myocardial infarction in patients presenting to emergency department with chest pain. Clin Biochem 2015;48:288-91.

26. McQueen MJ, Kavsak PA, Xu L, et al. Predicting myocardial infarction and other serious cardiac outcomes using high-sensitivity cardiac troponin $\mathrm{T}$ in a highrisk stable population. Clin Biochem 2013;46:5-9.

27. Omland T, Pfeffer MA, Solomon SD, et al. Prognostic value of cardiac troponin I measured with a highly sensitive assay in patients with stable coronary artery disease. J Am Coll Cardiol 2013;61:1240-9.

28. Kavsak PA, Wang X, Ko DT, et al. Short- and long-term risk stratification using a next-generation, high-sensitivity research cardiac troponin I (hs-cTnl) assay in an emergency department chest pain population. Clin Chem 2009;55:1809-15.

29. Kavsak PA, Beattie J, Pickersgill R, et al. A practical approach for the validation and clinical implementation of a high-sensitivity cardiac troponin I assay across a North American city. Pract Lab Med 2015;1:28-34.

30. Pickering JW, Greenslade JH, Cullen L, et al. Validation of presentation and $3 \mathrm{~h}$ high-sensitivity troponin to rule-in and rule-out acute myocardial infarction. Heart 2016;102:1270-8.

31. Keller T, Zeller T, Ojeda F, et al. Serial changes in highly sensitive troponin I assay and early diagnosis of myocardial infarction. JAMA 2011;306:2684-93.

32. Collinson PO, Heung YM, Gaze D, et al. Influence of population selection on the 99th percentile reference value for cardiac troponin assays. Clin Chem 2012;58:219-25.

33. Kavsak PA, Worster A, Shortt C, et al. Performance of high-sensitivity cardiac troponin in the emergency department for myocardial infarction and a composite cardiac outcome across different estimated glomerular filtration rates. Clin Chim Acta 2018;479:166-70. 
34. Andruchow JE, Kavsak PA, McRae AD. Contemporary emergency department management of patients with chest pain: a concise review and guide for the high-sensitivity troponin era. Can J Cardiol 2018;34:98-108.

35. Mueller-Hennessen M, Mueller C, Giannitsis E, et al. TRAPID-AMI Investigators. Serial sampling of high-sensitivity cardiac troponin $\mathrm{T}$ may not be required for prediction of acute myocardial infarction diagnosis in chest pain patients with highly abnormal concentrations at presentation. Clin Chem 2017;63:542-51.

36. Kavsak PA, Clark L, Jaffe AS. Effect of repeat measurements of high-sensitivity cardiac troponin on the same sample using the European Society of Cardiology 0-hour/1-hour or 2-hour algorithms for early rule-out and rule-in for myocardial infarction. Clin Chem 2017;63:1163-5.
37. Kavsak PA. External quality assessment testing near the limit of detection for high-sensitivity cardiac troponin assays. Clin Chem 2018 June 29 [Epub ahead of print]. doi:10.1373/clinchem.2018.288613.

38. Kavsak PA, Don-Wauchope AC, Hill SA, et al. Acceptable analytical variation may exceed high-sensitivity cardiac troponin I cutoffs in early rule-out and rule-in acute myocardial infarction algorithms. Clin Chem 2016;62:887-9.

39. Kavsak PA, Malinowski $P$, Roy $C$, et al. Assessing matrix, interferences and comparability between the Abbott Diagnostics and the Beckman Coulter high-sensitivity cardiac troponin I assays. Clin Chem Lab Med 2018;56: 1176-81.
Competing interests: Peter Kavsak has received research grants from the Canadian Institutes of Health Research (CIHR), Abbott Laboratories, Abbott Point of Care, Beckman Coulter, Randox Laboratories, Roche Diagnostics and Siemens Healthcare Diagnostics. He has received consultant or advisor fees, or honoraria from Abbott Laboratories, Abbott Point of Care, Abbott Diagnostics Division of Canada, Beckman Coulter, Randox Laboratories, Roche Diagnostics, Siemens Healthcare Diagnostics and Ortho Clinical Diagnostics. In addition, he has a pending patent application filed by McMaster University on a laboratory score for risk stratification in patients with possible cardiac injury. Johannes Neumann has received research grants from the German Heart Foundation/German Foundation of Heart Research and from Else Kröner Fresenius Stiftung. Louise Cullen has received grants from Abbott Diagnostics, Roche and Alere, and personal fees for education and consulting from Abbott Diagnostics, Siemens, Beckman Coulter and Alere. Martin Than has received grants and personal fees in the form of speaker fees or funding for education from Abbott, Alere, Beckman Coulter and Roche. Colleen Shortt is employed by Abbott Diagnostics. Jaimi Greenslade has received research grants that were provided to his institution from Roche and Abbott, as well as a research grant from Emergency Medicine Foundation to support data collection for this study. John Pickering has received nonfinancial support from Abbott Diagnostics and Roche Diagnostics outside the submitted work. Dirk Westermann has received speaker fees from Bayer, Boehringer Ingelheim, BerlinChemie, AstraZeneca, BIOTRONIK and
Novartis, outside the submitted work. William Parsonage has received research grants from Abbott Laboratories and Roche during the conduct of the study, and fees for being an advisory board member and nonfinancial support from Abbott Laboratories, outside the submitted work. P.J. Devereaux reports grants from Abbott Diagnostics, Boehringer Ingelheim, Covidien, Octopharma, Philips Healthcare, Roche Diagnostics and Stryker, outside the submitted work. Richard Troughton has received research grants from the Health Research Council of New Zealand, Roche Diagnostics and Merck, as well as consulting fees from Merck. Stefan Blankenberg has received honoraria from Abbott Diagnostics, Siemens, Thermo Fisher Scientific and Roche Diagnostics, outside the submitted work. Andrew Worster has received a research grant from $\mathrm{CIHR}$. In addition, he has a pending patent application filed by McMaster University on a laboratory score for risk stratification for patients with possible cardiac injury. No other competing interests were declared.

This article has been peer reviewed.

Affiliations: Departments of Pathology and Molecular Medicine (Kavsak, Hill, McQueen), Health Research Methods, Evidence and Impact (Ma, Griffith); and Medicine (Clayton); Division of Emergency Medicine (Shortt, Sherbino, Worster); Division of Cardiology (Mehta, Devereaux); Population Health Research Institute (Devereaux), McMaster University, Hamilton, Ont.; Department of General and Interventional Cardiology (Neumann, Ojeda, Westermann, Sörensen, Blankenberg), University Heart Center Hamburg Eppendorf, Hamburg, Germany; Royal Brisbane and
Women's Hospital (Cullen, Greenslade, Parsonage), Brisbane, Australia; Christchurch Hospital (Than, Pickering, Troughton, Aldous), Christchurch, New Zealand; Department of Medicine and Christchurch Heart Institute, University of Otago (Richards, Pickering, Troughton, Pemberton), Christchurch, New Zealand; Cardiovascular Research Institute (Richards), National University of Singapore

Contributors: Peter Kavsak, Andrew Worster, Louise Cullen, Martin Than and Stefan Blankenberg were responsible for the concept and design of the work. Johannes Neumann, Louise Cullen, Martin Than, Colleen Shortt, Jaimi Greenslade, John Pickering, Francisco Ojeda, Jinhui Ma, Natasha Clayton, Jonathan Sherbino, Stephen Hill, Matthew McQueen, Dirk Westermann, Nils Sörensen, William Parsonage, Lauren Griffith, Shamir Mehta, P.J. Devereaux, Mark Richards, Richard Troughton, Chris Pemberton, Sally Aldous, and Stefan Blankenberg critically revised the manuscript for important intellectual content. All of the authors acquired, analyzed or interpreted the data, gave final approval of the version to be published and agreed to be accountable for all aspects of the work.

Funding: This study was supported by a Canadian Institutes of Health Research grant, with reagent support from Abbott Laboratories and Roche Diagnostics.

Data sharing: The studies were conducted before data sharing processes were in place, and thus individual data are not available.

Accepted: July 9, 2018

Correspondence to: Peter Kavsak, kavsakp@mcmaster.ca 